Bull. Austral. Math. Soc.

$43 \mathrm{~A} 15,43 \mathrm{~A} 45,46 \mathrm{~J} 20$

VOL. 51 (1995) [33-42]

\title{
POLYNOMIALS AND FUNCTIONS WITH FINITE SPECTRA ON LOCALLY COMPACT ABELIAN GROUPS
}

\author{
B. Basit and A.J. Pryde
}

In this paper we define polynomials on a locally compact Abelian group $G$ and prove the equivalence of our definition with that of Domar. We explore the properties of polynomials and determine their spectra. We also characterise the primary ideals of certain Beurling algebras $L_{w}^{1}(\mathbb{Z})$ on the group of integers $\mathbb{Z}$. This allows us to classify those elements of $L_{w}^{\infty}(G)$ that have finite spectrum. If $\varphi$ is a uniformly continuous function with bounded differences then there is a Beurling algebra naturally associated with $\varphi$. We give a condition on the spectrum of $\varphi$ relative to this algebra which ensures that $\varphi$ is bounded. Finally we give spectral conditions on a bounded function on $\mathbb{R}$ that ensure that its indefinite integral is bounded.

\section{INTRODUCTION, NOTATION AND PRELIMINARIES}

Throughout this paper $G$ will denote a locally compact Abelian group equipped with Haar measure. We consider certain unbounded functions $\varphi: G \rightarrow \mathbb{C}$ and discuss the notion of Beurling spectrum for $\varphi$.

Firstly, the spaces $L^{p}(G)$ with corresponding norms $\|\cdot\|_{p}$ are defined as usual using the Haar measure (see [6]). We shall always identify functions on $G$ which agree almost everywhere (see $\left[3\right.$, p.36]). In particular, $L^{1}(G)$ is a Banach algebra under the convolution product and carries an involution defined by

$$
f^{*}(x)=\overline{f(-x)} \quad \text { for } \quad x \in G, \quad f \in L^{1}(G)
$$

By $L_{\text {loc }}^{\infty}(G)$ we mean the space of functions $\varphi: G \rightarrow \mathbb{C}$ such that $\chi_{K} \varphi \in L^{\infty}(G)$ for every compact set $K \subset G, \chi_{K}$ denoting the characteristic function of $K$.

The action of the dual group $\widehat{G}$ on $G$ will be denoted $\gamma(x)=(x, \gamma)$ where $x \in G$, $\gamma \in \widehat{G}$ and the Fourier transform of $f \in L^{1}(G)$ is defined by $\widehat{f}(\gamma)=\int_{G} f(x)(-x, \gamma) d x$ for $\gamma \in \widehat{G}$. Unless otherwise stated, the group operation on $G$ will be addition and on $\widehat{G}$ multiplication. The unit character in $\widehat{G}$ is denoted by $\widehat{e}$. The translate $\varphi_{y}$ and difference $\Delta_{y} \varphi$ by $y \in G$ of a function $\varphi: G \rightarrow \mathbb{C}$ are defined by

$$
\varphi_{y}(x)=\varphi(x+y) \text { and } \Delta_{y} \varphi(x)=\varphi(x+y)-\varphi(x) \text { for } x \in G .
$$

Received 7th March, 1994

Copyright Clearance Centre, Inc. Serial-fee code: 0004-9729/95 \$A2.00+0.00. 
Following Reiter [5, p.83] we call a function $w \in L_{l o c}^{\infty}(G)$ a weight function on $G$ if for all $x, y \in G$

$$
\begin{aligned}
w(x) & \geqslant 1 \\
w(x+y) & \leqslant w(x) w(y)
\end{aligned}
$$

Given a weight on $G$ we define

$$
L_{w}^{1}(G)=\left\{f \in L^{1}(G):\|f\|_{1, w}=\int_{G}|f(x)| w(x) d x<\infty\right\}
$$

Then $L_{w}^{1}(G)$ is a subalgebra of $L^{1}(G)$ which is a Banach algebra under the norm $\|\cdot\|_{1, w}$. The Banach space dual of $L_{w}^{1}(G)$ is

$$
L_{w}^{\infty}(G)=\left\{\varphi \in L_{l o c}^{\infty}(G):\|\varphi\|_{\infty, w}=\underset{x \in G}{\left.\operatorname{ess} \sup _{x} \frac{|\varphi(x)|}{w(x)}<\infty\right\}}\right.
$$

As noted by Reiter [5, p.84], we may assume that $w$ is upper semicontinuous, and so each continuous function $\varphi \in L_{w}^{\infty}(G)$ satisfies

$$
|\varphi(x)| \leqslant\|\varphi\|_{\infty, w} w(x) \quad \text { for all } x \in G
$$

It is known that $L_{w}^{1}(G)$ is a Wiener algebra if and only if $w$ satisfies the following condition of Beurling-Domar [5, p.132], [1, Theorem 2.11]:

$$
\sum_{m=1}^{\infty} \frac{\log w(m x)}{m^{2}}<\infty \text { for all } x \in G
$$

These algebras were introduced by Beurling for the case $G=\mathbb{R}$ and studied in the general case by Domar (see [1]).

If $\varphi \in L_{w}^{\infty}(G), f^{*} * \varphi$ is well-defined for all $f \in L_{w}^{1}(G)$ and $I_{w}(\varphi)=\left\{f \in L_{w}^{1}(G)\right.$ : $\left.f^{*} * \varphi=0\right\}$ is an ideal of the algebra $L_{w}^{1}(G)$. We define the Beurling spectrum of $\varphi$ relative to $L_{w}^{1}(G)$ to be the set

$$
s p_{w}(\varphi)=\text { hull } I_{w}(\varphi)=\left\{\gamma \in \widehat{G}: \widehat{f}(\gamma)=0 \text { for all } f \in I_{w}(\varphi)\right\}
$$

As each such function $\widehat{f}$ is continuous on $\widehat{G}, s p_{w}(\varphi)$ is a closed subset of $\widehat{G}$.

If in addition $w$ is bounded, so that $L_{w}^{1}(G)=L^{1}(G)$, we shall write $I(\varphi)=I_{w}(\varphi)$ and $s p(\varphi)=s p_{w}(\varphi)$. 
Proposition 1.1. Let $w$ be a weight function on $G$ satisfying the condition (1.8) and let $\varphi \in L_{w}^{\infty}(G)$.

(a) $s p_{w}\left(f^{*} * \varphi\right) \subset \operatorname{supp} \hat{f} \cap s p_{w}(\varphi)$ for all $f \in L_{w}^{1}(G)$.

(b) Given a neighbourhood $V$ of a compact set $W$ in $\widehat{G}$, there exists $f \in$ $L_{v}^{1}(G)$ such that $\widehat{f}=1$ on $W$ and $\operatorname{supp} \widehat{f} \subset V$.

(c) If $f \in L_{w}^{1}(G)$ and $\hat{f}=1$ on a neighbourhood of $\operatorname{sp}_{w}(\varphi)$ then $f^{*} * \varphi=\varphi$.

(d) $s p_{w}(\varphi)=\emptyset$ if and only if $\varphi=0$ and $s p_{w}(\varphi)=\{\hat{e}\}$ if $\varphi$ is a non-zero constant.

(e) $s p_{w}(\gamma \varphi)=\gamma s p_{w}(\varphi)$ for all $\gamma \in \widehat{G}$.

(f) $s p_{w}\left(\Delta_{y} \varphi\right) \subset s p_{w}(\varphi) \subset\{\hat{e}\} \bigcup_{z \in G} s p_{w}\left(\Delta_{z} \varphi\right)$ for all $y \in G$.

Proof: Since $L_{w}^{1}(G)$ is a Wiener algebra the proof of (a)-(e) can be carried out in the same manner as for $L^{1}(G)$. See $[2$, p.988] or [5, pp.140-141].

Let $y \in G$. Since $L_{w}^{1}(G)$ is translation invariant, $I_{w}(\varphi) \subset I_{w}\left(\Delta_{y} \varphi\right)$. Hence $s p_{w}\left(\Delta_{y} \varphi\right) \subset s p_{w}(\varphi)$. Conversely, suppose $\gamma \notin s p_{w}\left(\Delta_{z} \varphi\right)$ for all $z \in G$, and $\gamma \neq \widehat{e}$. Then there exists $z \in G$ such that $\gamma(z) \neq 1$ and $f \in I_{w}\left(\Delta_{z} \varphi\right)$ such that $\widehat{f}(\gamma) \neq 0$. If $g=\Delta_{-z} f$ then $\hat{g}(\gamma)=(\gamma(-z)-1) \hat{f}(\gamma) \neq 0$ whereas $g^{*} * \varphi=f^{*} * \Delta_{z} \varphi=0$. So $g \in I_{w}(\varphi)$ which implies $\gamma \notin s p_{w}(\varphi)$. This proves (f).

REMARK 1.2. Let $w_{1}$ and $w_{2}$ be two weight functions on $G$ satisfying the condition (1.8). If $\varphi \in L_{w_{1}}^{\infty}(G) \cap L_{w_{2}}^{\infty}(G)$ then $s p_{w_{1}}(\varphi)=s p_{w_{2}}(\varphi)$. Indeed, $w=w_{1} w_{2}$ is also a weight function on $G$ satisfying (1.8). Since $w_{1} \leqslant w$ and $w_{2} \leqslant w$ it follows from [5, p.138] that $s p_{w_{1}}(\varphi)=s p_{w}(\varphi)=s p_{w_{2}}(\varphi)$. If $\varphi \in L^{\infty}(G)$ and $w$ is a weight function satisfying (1.8), then $\varphi \in L_{w}^{\infty}(G)$ and so $s p_{w}(\varphi)=s p(\varphi)$.

\section{POLYNOMIALS AND THEIR SPECTRA}

In this section we define polynomials on $G$ and prove the equivalence of our definition with that of Domar [1, Definition 2.3.2]. We explore the properties of polynomials, and prove in particular that $s p_{w}(p)=\{\widehat{e}\}$ for each non-zero polynomial $p \in L_{w}^{\infty}(G)$.

A continuous function $\varphi: G \rightarrow \mathbb{C}$ will be called a polynomial of degree $n$ if

$$
\begin{array}{r}
\Delta_{y_{1}} \ldots \Delta_{y_{n+1}} \varphi=0 \text { for all } y_{1}, \ldots, y_{n+1} \in G \\
\Delta_{y_{1}} \ldots \Delta_{y_{n}} \varphi \neq 0 \text { for some } y_{1}, \ldots, y_{n} \in G .
\end{array}
$$

Denote by $\mathbb{Z}_{+}$the semigroup of non-negative integers with the discrete topology. Set $\mathbb{Z}_{+}^{k}=\left(\mathbb{Z}_{+}\right)^{k}, k \geqslant 1$. For arbitrary topological semigroups $H$ denote by $C(H)$ the set of continuous complex valued functions on $H$. The following two propositions will be found useful. The first is proved by induction and the second, a simple chain rule for difference operators, is obvious. 
Proposition 2.1. If $\varphi \in C(G)$ then $\varphi(x+m y)=\sum_{j=0}^{m}\left(\begin{array}{c}m \\ j\end{array}\right) \Delta_{y}^{j} \varphi(x)$ for all $m \in \mathbb{Z}_{+}$and $x, y \in G$.

Proposition 2.2. Given $\Phi \in C\left(G^{n}\right)$, define $\Psi \in C\left(\mathbb{Z}_{+}^{n} \times G^{n}\right)$ by $\Psi\left(t_{1}, \ldots, t_{n}, y_{1}, \ldots, y_{n}\right)=\Phi\left(t_{1} y_{1}, \ldots, t_{n} y_{n}\right)$, where $t_{j} \in \mathbb{Z}_{+}$and $y_{j} \in G$. If $\Delta_{y, j}$ denotes difference by $y$ in position $j$ only, then $\Delta_{y_{j}, j} \Phi\left(t_{1} y_{1}, \ldots, t_{n} y_{n}\right)=$ $\Delta_{1, j} \Psi\left(t_{1}, \ldots, t_{n}, y_{1}, \ldots, y_{n}\right)$.

Recall that a function $\varphi \in C(G)$ is a polynomial of degree $\mathrm{n}$ in the sense of Domar if $\varphi(x+t y)$ is a polynomial in $t \in \mathbb{Z}_{+}$of degree at most $\mathrm{n}$ for each $x, y \in G$ and of degree $\mathrm{n}$ for some $x, y \in G$.

Proposition 2.3. For a function $\varphi \in C(G)$ the following are equivalent:

(a) $\Delta_{y_{1}} \ldots \Delta_{y_{n+1}} \varphi=0$ for all $y_{1}, \ldots y_{n+1} \in G$.

(b) $\Delta_{y}^{n+1} \varphi=0$ for all $y \in G$.

(c) $\varphi(x+t y)$ is a polynomial in $t \in \mathbb{Z}_{+}$of degree at most $n$ for all $x, y \in G$.

(d) $\varphi\left(x+t_{1} y_{1}+\ldots+t_{k} y_{k}\right)$ is a polynomial in $\left(t_{1}, \ldots, t_{k}\right) \in \mathbb{Z}_{+}^{k}$ of degree at most $n$ for all $x, y_{1}, \ldots, y_{k} \in G$, and $k>0$.

Proof: That (a) implies (b) is obvious. If (b) holds then by Proposition 2.1 we have $\varphi(x+t y)=\sum_{j=0}^{n}\left(\begin{array}{l}t \\ j\end{array}\right) \Delta_{y}^{j} \varphi(x)$, showing that (b) implies (c). Given (c) and $x, y_{1}, \ldots, y_{k} \in G$, define $\psi(t)=\varphi\left(x+t_{1} y_{1}+\ldots+t_{k} y_{k}\right)$ for $\left(t_{1}, \ldots, t_{k}\right) \in \mathbb{Z}_{+}^{k}$. Then $\psi$ is a polynomial in each $t_{j}$ of degree at most $\mathbf{n}$. So $\psi$ is a polynomial in $t$ of degree at most kn. Hence

$$
\psi(t)=\sum_{|\alpha| \leqslant k n} a_{\alpha} t^{\alpha}, \text { where } a_{\alpha} \in \mathbb{C}, \alpha=\left(\alpha_{1}, \ldots, \alpha_{k}\right) \in \mathbb{Z}_{+}^{k} \text { and }|\alpha|=\alpha_{1}+\ldots+\alpha_{k}
$$

Moreover, for $\lambda \in \mathbb{Z}_{+}$, the function

$$
\psi(\lambda t)=\sum_{|\alpha| \leqslant k n} a_{\alpha} \lambda^{|\alpha|} t^{\alpha}=\varphi\left(x+\lambda t_{1} y_{1}+\ldots+\lambda t_{k} y_{k}\right)
$$

is again a polynomial in $\lambda$ of degree at most $\mathrm{n}$. Hence $a_{\alpha}=0$ for $|\alpha|>n$, showing that (c) implies (d). Finally, given (d) and $x, y_{1}, \ldots, y_{n+1} \in G$, define $\psi(t)=\varphi\left(x+t_{1} y_{1}+\cdots+t_{n+1} y_{n+1}\right)$ for $\left(t_{1}, \ldots, t_{n+1}\right) \in \mathbb{Z}_{+}^{n+1}$. Then $\psi$ is a polynomial in $t$ of degree at most $n$. So all $(n+1)$ th differences of $\psi$ are zero. Hence, by Proposition 2.2, $\Delta_{y_{1}} \ldots \Delta_{y_{n+1}} \varphi(x)=\Delta_{1,1} \ldots \Delta_{1, n+1} \psi(0)=0$, showing that (d) implies (a).

THEOREM 2.4. For a function $\varphi \in C(G)$ the following are equivalent:

(a) $\varphi$ is a polynomial of degree $n$. 
(b) $\Delta_{y}^{n+1} \varphi=0$ for all $y \in G$ and $\Delta_{y}^{n} \varphi \neq 0$ for some $y \in G$.

(c) $\varphi$ is a polynomial of degree $n$ in the sense of Domar.

(d) $\varphi\left(x+t_{1} y_{1}+\ldots+t_{k} y_{k}\right)$ is a polynomial in $\left(t_{1}, \ldots, t_{k}\right) \in \mathbb{Z}_{+}^{k}$ of degree at most $n$ for all $x, y_{1}, \ldots, y_{k} \in G, k>0$ and of degree $n$ for some $x, y_{1}, \ldots, y_{k} \in G, k>0$.

Proof: Given (d), we have Proposition 2.3(c). Moreover we can choose $x, y_{1}, \ldots, y_{k}$ such that $\varphi\left(x+t_{1} y_{1}+\ldots+t_{k} y_{k}\right)$ is a polynomial in $\left(t_{1}, \ldots, t_{k}\right) \in \mathbb{Z}_{+}^{k}$ of degree n. So $\varphi\left(x+t_{1} y_{1}+\ldots+t_{k} y_{k}\right)=\sum_{|\alpha| \leqslant n} a_{\alpha} t^{\alpha}$, where $a_{\alpha} \in \mathbb{C}$, and $\sum_{|\alpha|=n} a_{\alpha} t^{\alpha}$ is not identically zero. Choose $t$ so that $\sum_{|\alpha|=n} a_{\alpha} t^{\alpha} \neq 0$ and set $y=t_{1} y_{1}+\ldots+t_{k} y_{k}$. Then $\varphi(x+\lambda y)=\sum_{|\alpha| \leqslant n} a_{\alpha} t^{\alpha} \lambda^{|\alpha|}$ is a polynomial in $\lambda \in \mathbb{Z}_{+}$of degree $\mathrm{n}$, showing that (d) implies (c). Given(c), we have Proposition 2.3(b). Moreover, there exist $x, y \in G$ such that $\varphi(x+t y)$ is of degree $\mathrm{n}$ in $t \in Z_{+}$. So $\varphi(x+t y)=\sum_{j=0}^{n} a_{j} t^{j}$ where $a_{j} \in \mathbb{C}, a_{n} \neq 0$. By Proposition 2.2, $\Delta_{y}^{n} \varphi(x)=\sum_{j=0}^{n} a_{j} \Delta_{1}^{n} t^{j}=a_{n} n ! \neq 0$, showing that (c) implies (b). That (b) implies (a) is obvious. Finally, if (a) holds then Proposition 2.3(d) holds. Moreover, there exist $x, y_{1}, \ldots, y_{n} \in G$ such that $\Delta_{y_{1}} \ldots \Delta_{y_{n}} \varphi(x) \neq 0$. So $\varphi\left(x+t_{1} y_{1}+\ldots+t_{n} y_{n}\right)=\sum_{|\alpha| \leqslant n} a_{\alpha} t^{\alpha}$ and by Proposition $2.2, \Delta_{y_{1}} \ldots \Delta_{y_{n}} \varphi(x)=a_{\beta}$ where $\beta=(1,1, \ldots, 1) \in \mathbb{Z}_{+}^{n}$. So $a_{\beta} \neq 0$ and $\varphi\left(x+t_{1} y_{1}+\ldots+t_{n} y_{n}\right)$ is a polynomial in $\left(t_{1}, \ldots, t_{n}\right)$ of degree $n$, showing that (a) implies (d).

REMARKS 2.5. (a) On any Abelian group $G$ the polynomials of degree 0 are precisely the constant functions. Polynomials of degree larger than 0 are unbounded. So the only polynomials on a compact group are the constant functions. Any polynomial $\varphi$ on $\mathrm{G}$ of degree 1 is of the form $\varphi(x)=\varphi(0)+\alpha(x)$, where $\alpha(x+y)=\alpha(x)+\alpha(y)$ for $x, y \in G$.

(b) The definition of polynomials and the results proved so far in this section require only that $\mathrm{G}$ be an Abelian topological semigroup.

We turn now to the investigation of spectra of polynomials.

Proposition 2.6. Let $p$ be a non-zero polynomial on G. Suppose that $w$ is a weight function satisfying (1.8) and $p \in L_{w}^{\infty}(G)$. Then $s p_{w}(\gamma p)=\{\gamma\}$ for all $\gamma \in \widehat{G}$. In particular $s p_{w}(p)=\{\hat{e}\}$

Proof: Let $\mathrm{p}$ have degree $\mathrm{n}$. By Theorem 2.4, $\Delta_{y_{0}}^{n} p \neq 0$ for some $y_{0} \in G$. Applying Proposition 1.1(f) n times we obtain $s p_{w}\left(\Delta_{y_{0}}^{n} p\right) \subset s p_{w}(p) \subset\{\widehat{e}\} \bigcup_{y \in G} s p_{w}\left(\Delta_{y}^{n} p\right)$. But by Proposition $1.1(\mathrm{~d}), s p_{w}\left(\Delta_{y_{0}}^{n} p\right)=\{\hat{e}\}$ and $s p_{w}\left(\Delta_{y}^{n} p\right) \subset\{\hat{e}\}$ for each $y \in G$. 
Hence $s p_{w}(p)=\{\widehat{e}\}$ and by Proposition 1.1(e), $s p_{w}(\gamma p)=\{\gamma\}$.

\section{PRIMARY IDEALS AND FunCtions With FINITE SPECTRUM}

In this section we study Beurling algebras $L_{w}^{1}(G)$ when the weight function $w$ satisfies the following two conditions for some $N \in \mathbb{Z}_{+}$:

$$
\begin{gathered}
\sup _{m \in \mathbf{Z}} \frac{w(m x)}{1+|m|^{N+\alpha(x)}}<\infty \text { for all } x \in G \text { and some } \alpha(x)<1 ; \\
\inf _{m \in \mathbf{Z}} \frac{w(m x)}{|m|^{N}}>0 \text { for some } x \in G .
\end{gathered}
$$

It should be noted that condition (3.1) implies the Beurling-Domar condition (1.8) Using conditions (3.1) and (3.2) we characterise those functions $\varphi \in L_{w}^{\infty}(G)$ for which $s p_{w}(\varphi)$ is finite.

We begin by studying the primary ideals of $L_{w}^{1}(\mathbb{Z})$ when $w$ satisfies (3.1) and (3.2) with $G=\mathbb{Z}$. Recall that a primary ideal is one that is contained in exactly one maximal ideal, and the cospectrum of an ideal J of $L_{w}^{1}(G)$ is the set $\{\gamma \in \widehat{G}: \widehat{f}(\gamma)=$ 0 for all $f \in J\}$.

When $G=\mathbb{Z}$, conditions (3.1) and (3.2) are equivalent to the existence of $c_{1}>$ $0, c_{2}>0$ and $\alpha \in(0,1)$ such that

$$
c_{1}\left(1+|m|^{N}\right) \leqslant w(m) \leqslant c_{2}\left(1+|m|^{N+\alpha}\right) \text { for all } m \in \mathbb{Z} .
$$

In this case, $\left\{\widehat{f}: f \in L_{w}^{1}(\mathbb{Z})\right\}$ is a subalgebra of $C^{N}(T)$, the algebra of continuous functions on $T=\mathbb{R} / 2 \pi \mathbb{Z}$ with derivatives up to order $\mathrm{N}$ also continuous on $T$. We then define

$$
I_{k}=\left\{f \in L_{w}^{1}(\mathbb{Z}): \widehat{f}^{(j)}(\widehat{e})=0 \text { for } 0 \leqslant j \leqslant k\right\}, \text { where } 0 \leqslant k \leqslant N .
$$

So $I_{k}$ is a closed primary ideal of $L_{w}^{1}(\mathbb{Z})$ with cospectrum $\{\hat{e}\}$.

ThEOREM 3.1. Let $w$ be a weight function on $\mathbb{Z}$ satisfying (3.3). Then $I_{0}, I_{1}, \ldots, I_{N}$ are the only closed primary ideals of $L_{w}^{1}(\mathbb{Z})$ with cospectrum $\{\hat{e}\}$.

PROOF: Let $e_{m}(n)=\delta_{m, n}$ denote the Kronecker function, where $m, n \in \mathbb{Z}$. Then $e_{1}$ and $e_{-1}$ generate the algebra $L_{w}^{1}(\mathbb{Z})$, and $\widehat{e_{1}}(t)=e^{i t}$ and $\widehat{e_{-1}}(t)=e^{-i t}$ for $t \in T$. Moreover, $e_{1}^{m}=e_{m}$ so $\alpha_{m}=\left\|e_{1}^{m}\right\|_{1, w}=w(m)$ for each $m \in \mathbb{Z}$. It follows from (3.3) that $\lim _{r \rightarrow 1-}(1-r)^{N+2} \sum_{m=0}^{\infty} \alpha_{m} r^{m}=\lim _{r \rightarrow 1-}(1-r)^{N+2} \sum_{m=0}^{\infty} \alpha_{-m} r^{-m}=0$. By a theorem of Gelfand [4, p.209], $L_{w}^{1}(\mathbb{Z})$ has exactly $N+1$ ideals with cospectrum $\{\hat{e}\}$, namely $I_{0}, I_{1}, \ldots, I_{N}$. 
Proposition 3.2. Let $w$ be a weight function on $\mathbb{Z}$ satisfying (3.3). If $0 \leqslant$ $k \leqslant N$ then $\Delta_{m}^{k+1} f \in I_{k}$ for all $m \in \mathbb{Z}$ and all $f \in L_{w}^{1}(\mathbb{Z})$.

PROOF: If $g=\Delta_{m}^{k+1} f$ then $\widehat{g}(t)=\left(e^{i t m}-1\right)^{k+1} \widehat{f}(t)$. Hence $g \in I_{k}$ as claimed. $]$

COROLlary 3.3. Let $w$ be a weight function on $\mathbb{Z}$ satisfying (3.3). If $\varphi \in$ $L_{w}^{1}(\mathbb{Z})$ and $s p_{w}(\varphi)=\{\widehat{e}\}$, then $\varphi$ is a polynomial of degree at most $N$.

Proof: Since $I_{w}(\varphi)$ is a closed ideal with cospectrum $\{\hat{e}\}$, we conclude from Theorem 3.1 that $I_{w}(\varphi)=I_{k}$ for some $\mathrm{k}, 0 \leqslant k \leqslant N$. Now for arbitrary $f \in L_{w}^{1}(\mathbb{Z})$ and $m \in \mathbb{Z}$, we have $\Delta_{-m}^{k+1} f \in I_{k}$ and so $f^{*} * \Delta_{m}^{k+1} \varphi=\left(\Delta_{-m}^{k+1} f\right)^{*} * \varphi=0$. So $I_{w}\left(\Delta_{m}^{k+1} \varphi\right)=L_{w}^{1}(\mathbb{Z})$ and $s p_{w}\left(\Delta_{m}^{k+1} \varphi\right)=\emptyset$. By Proposition $1.1(\mathrm{~d}), \Delta_{m}^{k+1} \varphi=0$, and by Proposition 2.3, $\varphi$ is a polynomial of degree at most $\mathbf{k}$.

We now consider functions on more general groups $\mathrm{G}$.

THEOREM 3.4. Let $w$ be a weight function satisfying (3.1) and (3.2) and let $\varphi \in L_{w}^{\infty}(G)$. Let $\operatorname{sp}_{w}(\varphi)=\left\{\gamma_{1}, \ldots, \gamma_{n}\right\}$. Then there exist polynomials $p_{1}, \ldots, p_{n}$ in $L_{w}^{\infty}(G)$ of degree at most $N$ such that $\varphi=\sum_{k=1}^{n} \gamma_{k} p_{k}$. In particular if $s p_{w}(\varphi)=\{\widehat{e}\}$ then $\varphi=p$ for some polynomial $p$ of degree at most $N$.

PROOF: As $s p_{w}(\varphi)$ is compact, $\varphi$ coincides almost everywhere with a continuous function. (See $\left[5\right.$, p.142].) We may therefore assume $\varphi \in C(G)$. Now suppose $s p_{w}(\varphi)=$ $\{\hat{e}\}$. For $y \in G$, let $H_{y}$ be the subgroup generated by $y$ and let $K_{y}$ be its closure. Let $w_{y}$ be the restriction of $w$ to $K_{y}$ and $\varphi_{y}(h)=\varphi(h+y)$ for $h \in K_{y}$. Then $\varphi_{y} \in L_{w_{y}}^{\infty}\left(K_{y}\right)$ and by a theorem of Reiter (see [5, p.144]) $s p_{w_{y}}\left(\varphi_{y}\right) \subset \pi\left(s p_{w}(\varphi)\right)=$ $\left\{\widehat{e_{y}}\right\}$, where $\pi: \widehat{G} \rightarrow \widehat{K}_{y}$ is the canonical projection and $\widehat{e}_{y}$ is the unit in $\widehat{K}_{y}$. By a theorem of Weil (see $[5, \mathrm{p} .88]$ ) either $K_{y}$ is compact or $K_{y}$ is isomorphic to $\mathbb{Z}$ $\left(H_{y}=\mathbb{Z}\right)$. If $\mathrm{K}$ is compact then $\varphi$ is bounded with $\operatorname{sp}\left(\varphi_{y}\right) \subset\left\{\widehat{e}_{y}\right\}$. Therefore $\varphi_{y}$ is a constant. (See Rudin $[6,6.8 .3]$.) On the other hand, by Corollary 3.3 , if $\boldsymbol{H}_{\boldsymbol{y}}=\mathbb{Z}$ then $\varphi$ is a polynomial of degree at most $N$. Applying these results to $\psi$, defined by $\psi(z)=\varphi(x+z)$ for $x, z \in G$, and noting that $s p_{w}(\psi)=s p_{w}(\varphi)$ we conclude that $\varphi(x+m y)$ is a polynomial in $m \in \mathbb{Z}_{+}$of degree at most $\mathrm{N}$ for each $x, y \in G$. So $\varphi$ is a polynomial of degree at most $\mathrm{N}$. For the general case, $s p_{w}(\varphi)=\left\{\gamma_{1}, \ldots, \gamma_{n}\right\}$, choose a neighbourhood $\mathrm{V}$ of $\{\hat{e}\}$ in $\widehat{G}$ and functions $f_{1}, \ldots, f_{n} \in L_{w}^{1}(G)$ such that $\widehat{f}_{k}=1$ on $\gamma_{k} V$ and $\gamma_{j} V \cap \gamma_{k} V=\operatorname{supp} \widehat{f}_{j} \cap \operatorname{supp} \widehat{f}_{k}=\emptyset$ for $j, k=1, \ldots, n$ and $j \neq k$. (See Proposition 1.1(b).) Then $s p_{w}\left(f_{k}^{*} * \varphi\right) \subset \operatorname{supp} \hat{f}_{k} \cap s p_{w}(\varphi)=\left\{\gamma_{k}\right\}$ and by the first part of this proof, $\gamma_{k}^{-1}\left(f_{k}^{*} * \varphi\right)=p_{k}$, a polynomial of degree at most $\mathrm{N}$. So $f^{*} * \varphi=\sum_{k=1}^{n} \gamma_{k} p_{k}$, where $f=\sum_{k=1}^{n} f_{k}$. But $\hat{f}=1$ on a neighbourhood of $s p_{v}(\varphi)$ and so, again by Proposition 1.1, $f^{*} * \varphi=\varphi$. 


\section{UNIFORMLY CONTINUOUS FUNCTIONS WITH BOUNDED DIFFERENCES}

Let $C_{u}(G)$ be the space of uniformly continuous complex-valued functions on $\mathrm{G}$. Let $C_{u b}(G)=C_{u}(G) \cap L^{\infty}(G)$ and denote by $C_{u d}(G)$ the linear subspace of $C_{u}(G)$ consisting of functions $\varphi \in C_{u}(G)$ satisfying

$$
\Delta_{h} \varphi \in C_{u b}(G) \text { for all } h \in G \text {. }
$$

We notice that if $G$ is connected then by a theorem of Pontrjagin (see [5, p.97]) $G=K \times \mathbb{R}^{n}$, where $\mathrm{K}$ is compact and connected and $n \geqslant 0$. It follows that $C_{u d}(G)=$ $C_{u}(G)$.

In this section we study the spectra of functions $\varphi \in C_{u d}(G)$. To do this we construct associated Beurling algebras. Indeed, define $w_{\varphi}: G \rightarrow \mathbb{C}$ by

$$
w_{\varphi}(y)=1+\frac{1}{2}\left(\left\|\Delta_{y} \varphi\right\|_{\infty}+\left\|\Delta_{-y} \varphi\right\|_{\infty}\right), y \in G
$$

Proposition 4.1. If $\varphi \in C_{u d}(G)$ then $w_{\varphi}$ is a uniformly continuous weight function on $G$ satisfying the condition

$$
\lim _{|m| \rightarrow \infty} \frac{w_{\varphi}(m x)}{m^{2}}=0 \text { for all } x \in G .
$$

Moreover $\varphi \in L_{w_{\varphi}}^{\infty}(G)$.

Proof: Let $\alpha(y)=w_{\varphi}(y)-1$. Then $\alpha\left(y_{1}+y_{2}\right) \leqslant \alpha\left(y_{1}\right)+\alpha\left(y_{2}\right), \alpha(y)=\alpha(-y)$, and $\left|\alpha\left(y_{1}+y_{2}\right)-\alpha\left(y_{2}\right)\right| \leqslant \alpha\left(y_{1}\right)$ for $y, y_{1}, y_{2} \in G$. It follows that $\alpha$ and $w_{\varphi}$ are uniformly continuous on $G$, and that $w_{\varphi}$ is a weight function. Moreover, for $n \in \mathbb{Z}$ and $y \in G, w_{\varphi}(m y)=1+\alpha(m y) \leqslant 1+|m| \alpha(y)$, from which (4.3) follows. Finally, $|\varphi(x)| \leqslant|\varphi(x)-\varphi(0)|+|\varphi(0)| \leqslant 2 \alpha(x)+|\varphi(0)| \leqslant(2+|\varphi(0)|) w_{\varphi}(x)$, for $x \in G$, showing $\varphi \in L_{w_{\varphi}}^{\infty}(G)$.

We remark that condition (4.3) implies condition (3.1) with $\mathrm{N}=1$. This in turn implies the Beurling-Domar condition (1.8).

TheOREM 4.2. If $\varphi \in C_{u d}(G)$ and $\widehat{e} \notin s p_{w_{\varphi}}(\varphi)$ then $\varphi$ is bounded.

Proof: Let $V$ be a compact neighbourhood of $\widehat{e}$ such that $V \cap s p_{w_{\varphi}}(\varphi)=\emptyset$. By Proposition 1.1 there exists $f \in L_{w_{\varphi}}^{1}(G)$ such that $\operatorname{supp} \hat{f} \subset V$ and $\hat{f}(\widehat{e})=1$. So $s p_{w_{\varphi}}\left(f^{*} * \varphi\right)=\emptyset$ and hence $f^{*} * \varphi=0$. Hence $\varphi(t)=\varphi(t)-f^{*} * \varphi(t)=\int_{G}[\varphi(t)-$ $\varphi(t-s)] f^{*}(s) d s$ and $|\varphi(t)| \leqslant 2 \int_{G} w_{\varphi}(s)\left|f^{*}(s)\right| d s=2\|f\|_{1, w_{\varphi}}$, for $t \in G$. So $\varphi$ is bounded.

As is customary, we identify $\widehat{\mathbb{R}^{n}}$ with $\mathbb{R}^{n}$ so that $\widehat{\mathbb{R}}^{n}$ becomes an additive group with unit 0 . We now consider the indefinite integrals $P \varphi\left(x_{1}, x_{2}, \ldots, x_{n}\right)=$ $\int_{0}^{x_{1}} \varphi\left(t, x_{2}, \ldots, x_{n}\right) d t$ of functions $\varphi \in L^{\infty}\left(\mathbb{R}^{n}\right)$. 
Theorem 4.3. If $\varphi \in L^{\infty}\left(\mathbb{R}^{n}\right), 0 \notin s p(\varphi)$ and $P \varphi \in C_{u}\left(\mathbb{R}^{n}\right)$ then $P \varphi$ is bounded.

Proof: If $w\left(x_{1}, \ldots, x_{n}\right)=1+\left|x_{1}\right|$ then $w$ is a weight on $\mathbb{R}^{n}$ satisfying (1.8) and $\varphi, P \varphi \in L_{w}^{\infty}\left(\mathbb{R}^{n}\right)$. By remark 1.2, $s p(\varphi)=s p_{w}(\varphi)$ and similar to Proposition 1.1(f), $s p_{w}(P \varphi) \subset s p(\varphi) \cup\{0\}$. Let $V$ be a neighbourhood of 0 with $s p(\varphi) \cap V=$ $\emptyset$. By Proposition 1.1(b), there is a function $h \in L_{w}^{1}\left(\mathbb{R}^{n}\right)$ such that $\widehat{h}=1$ on a neighbourhood $\mathrm{W}$ of 0 and supp $\widehat{h} \subset V$. By Proposition 1.1 (a), sp $\left(h^{*} * P \varphi\right) \subset\{0\}$ and $h^{*} * \varphi=0$. Since $w$ also satisfies the conditions (3.1) and (3.2) with $N=1$, we have by Theorem $3.4, h^{*} * P \varphi=p$, a polynomial of degree at most 1 in $L_{w}^{\infty}\left(\mathbb{R}^{n}\right)$. Since, by (1.7) $|p(x)| \leqslant c\left(1+\left|x_{1}\right|\right)$ for some constant $c>0$ and all $x \in \mathbb{R}^{n}$, we conclude that $p(x)=h^{*} * P \varphi=c_{1}+c_{2} x_{1}$ for some $c_{1}, c_{2} \in \mathbb{C}$. But $\left(\partial\left(h^{*} * P \varphi\right)\right) /\left(\partial x_{1}\right)=h^{*} * \varphi=0$. Hence $c_{2}=0$ and $h^{*} * P \varphi=c_{1}$. If $\eta=P \varphi-c_{1}$ then $0 \notin s p_{v}(\eta)$. Indeed, $h^{*} * \eta=$ $h^{*} * P \varphi-h^{*} * c_{1}=c_{1}-c_{1}=0$. Thus $h \in I_{w}(\eta)$ and $\widehat{h}(0)=1$, showing $0 \notin s p_{w}(\eta)$. Finally, $\eta \in C_{u}\left(\mathbb{R}^{n}\right)=C_{u d}\left(\mathbb{R}^{n}\right)$ so by Theorem $4.2, \eta$ is bounded. Hence $P \varphi=\eta+c_{1}$ is bounded.

Corollary 4.4. If $\varphi \in L^{\infty}(\mathbb{R})$ and $0 \notin s p(\varphi)$ then $P \varphi$ is bounded.

Proof: Since $|P \varphi(x)-P \varphi(y)| \leqslant\|\varphi\|_{\infty}|x-y|, P \varphi \in C_{u}(\mathbb{R})$ and the corollary follows from the theorem.

Remark 4.5. Let $\varphi(x)=x e^{i x}$ and $w(x)=1+|x|$ for $x \in \mathbb{R}$. By Proposition 2.6 $s p_{w}(\varphi)=\{1\}$. So $0 \notin s p_{w}(\varphi)$ yet $\varphi$ is unbounded. Hence the condition $\varphi \in C_{u d}(G)$ is necessary in Theorem 4.2. Let $\varphi(x, y)=e^{i y}$ for $(x, y) \in \mathbb{R}^{2}$. Then $\operatorname{sp}(\varphi)=\{(0,1)\}$. So $(0,0) \notin s p(\varphi)$ yet $P \varphi(x, y)=x e^{i y}$ is unbounded. Hence the condition $P \varphi \in C_{u}(\mathbb{R})$ is necessary in Theorem 4.3.

\section{References}

[1] Y. Domar, 'Harmonic analysis based on certain Banach algebras', Acta Math. 96 (1956), 1-66.

[2] N. Dunford and J.T. Schwartz, Linear operators, Part II (Interscience Publishers, New York, London, 1963).

[3] L.H. Loomis, An introduction to harmonic nalysis (D. Van Nostrand Comp., Inc., 1953).

[4] M.A. Naimark, Normed rings, (translated from Russian) (P. Noordhoff, N.V. Groningen, The Netherlands, 1959).

[5] H. Reiter, Classical harmonic analysis and locally compact groups, Oxford Math. Monographs (Oxford University, 1968).

[6] W. Rudin, Fourier analysis on groups (Interscience, New York, London, 1962). 
Department of Mathematics

Monash University

Clayton Vic 3168

Australia

E-mail: bbasit@vaxc.cc.monash.edu.au

ajpryde@vaxc.cc.monash.edu.au 